\title{
Topotecan-Vincristine-Doxorubicin in Stage 4 High-Risk Neuroblastoma Patients Failing to Achieve a Complete Metastatic Response to Rapid COJEC: A SIOPEN Study
}

\section{Loredana Amoroso, $\mathrm{MD}^{1}$ \\ Giovanni Erminio, MD² \\ Guy Makin, MD ${ }^{3}$ \\ Andrew D. J. Pearson, MD ${ }^{4}$ \\ Penelope Brock ${ }^{5}$ \\ Dominique Valteau-Couanet, $\mathrm{MD}^{6}$ \\ Victoria Castel, $\mathrm{MD}^{7}$ \\ Marlène Pasquet, $\mathrm{MD}^{8}$ \\ Genevieve Laureys, $\mathrm{MD}^{9}$ \\ Caroline Thomas, MD10 \\ Roberto Luksch, MD11 \\ Ruth Ladenstein, MD'12 \\ Riccardo Haupt, MD2 \\ Alberto Garaventa, MD' \\ On behalf of SIOPEN Group}

*A list author's affiliations appears at the end of the paper.

\section{Purpose}

Metastatic response to induction therapy for high-risk neuroblastoma is a prognostic factor. In the International Society of Paediatric Oncology Europe Neuroblastoma (SIOPEN) HR-NBL-1 protocol, only patients with metastatic complete response (CR) or partial response (PR) with $\leq$ three abnormal skeletal areas on iodine 123-metaiodobenzylguanidine ([123] $] \mathrm{mIBG}$ ) scintigraphy and no bone marrow disease proceed to high dose therapy (HDT). In this study, topotecan-vincristine-doxorubicin (TVD) was evaluated in patients failing to achieve these criteria, with the aim of improving the metastatic response rate.

\section{Materials and Methods}

Patients with metastatic high-risk neuroblastoma who had not achieved the SIOPEN criteria for HDT after induction received two courses of topotecan $1.5 \mathrm{mg} / \mathrm{m}^{2} /$ day for 5 days, followed by a 48-hour infusion of vincristine, $2 \mathrm{mg} / \mathrm{m}^{2}$, and doxorubicin, $45 \mathrm{mg} / \mathrm{m}^{2}$.

Results

Sixty-three patients were eligible and evaluable. Following two courses of TVD, four (6.4\%) patients had an overall CR, while $28(44.4 \%)$ had a PR with a combined response rate of $50.8 \%$ (95\% confidence interval [CI], 37.9 to 63.6). Of these, 23 patients achieved a metastatic CR or a PR with $\leq 3$ mIBG skeletal areas and no bone marrow disease $(36.5 \%$; $95 \% \mathrm{Cl}, 24.7$ to 49.6 ) and were eligible to receive HDT. Toxicity was mostly haematological, affecting 106 of the 126 courses (84.1\%; 95\% Cl, 76.5 to 90.0), and dose reduction was necessary in six patients. Stomatitis was the second most common nonhematological toxicity, occurring in 20 patients (31.7\%).

\section{Conclusion}

TVD was effective in improving the response rate of high-risk neuroblastoma patients after induction with COJEC enabling them to proceed to HDT. However, the long-term benefits of TVD needs to be determined in randomized clinical trials.

\section{Key words}

Neuroblastoma, Recurrence, Child, Neoplasm,

Phase 2 clinical trial, Second line drugs
Correspondence: Riccardo Haupt, MD

Epidemiology and Biostatistics Unit,

Istituto Giannina Gaslini, Largo Gaslini 5,

16147 Genova, Italy

Tel: 39-01056363874

Fax: 39-0108981116

E-mail: riccardohaupt@gaslini.org

Received October 24, 2016

Accepted March 9, 2017

Published Online March 21, 2017 


\section{Introduction}

Stage 4 neuroblastoma in children $>18$ month of age is still a challenging disease to treat, despite modern therapeutic strategies, with two-thirds of patients refractory to first-line therapy or developing disease progression or relapse after an initial response [1,2].

Rapid COJEC (two courses of carboplatin, etoposide, vincristine; four courses of cisplatin, vincristine; two courses of etoposide, cyclophosphamide) is a time-intensive chemotherapy regimen administered at 10-day intervals that is utilised by the International Society of Paediatric Oncology Europe Neuroblastoma Group (SIOPEN) as the induction therapy for high-risk neuroblastoma patients (SIOPEN HR-NBL-1) [3,4]. Incomplete metastatic response to induction therapy for high-risk neuroblastoma has been shown to be associated with an inferior long-term outcome [5-7]. Therefore, SIOPEN elected for patients with high-risk neuroblastoma only proceeds to high dose therapy (HDT) if the patients have achieved a metastatic complete response (CR) or a "good" partial response (GPR). This is defined as at least a 50\% reduction in skeletal iodine 123-metaiodobenzylguanidine $\left(\left[{ }^{123} \mathrm{I}\right] \mathrm{mIBG}\right)$ positivity and $\leq 3$ abnormal skeletal areas on $\mathrm{mIBG}$ scintigraphy and no evidence of tumour on bone marrow aspirates and trephines after rapid COJEC. The status of the primary tumour is irrelevant to the decision to proceed to HDT. Given that with COJEC about $31 \%$ of patients do not achieve these strict SIOPEN criteria for HDT (Ladenstein $\mathrm{R}$ et al., personal communication), further strategies are needed to improve response and increase the number of patients becoming eligible for HDT.

In a previous phase II study of relapsed or refractory patients with neuroblastoma [8], the combination of topotecan given for 5 consecutive days prior to vincristine and doxorubicin administered simultaneously in a 48-hour continuous infusion (topotecan-vincristine-doxorubicin [TVD] regimen) showed a combined response rate (RR) of $64 \% \mathrm{CR}$ and partial response (PR).

Herein, we report the efficacy and toxicity of the TVD regimen (clinical trials identifier, NCT00392340) given to improve the metastatic response in children following induction therapy in SIOPEN HR-NBL-1, facilitating them to receive HDT with the lowest metastatic burden.

\section{Materials and Methods}

This was an open-label, multi-centre, phase II study included in the therapeutic strategy of SIOPEN HR-NBL-1 protocol designed to evaluate the efficacy and toxicity of two courses of TVD in patients with stage 4 high-risk neuroblastoma. Patients eligible for TVD were those in whom the best metastatic response after induction therapy with COJEC was a "poor" PR (PPR, defined as $<50 \%$ reduction in skeletal mIBG positivity and/or $>$ three areas of abnormal skeletal uptake on mIBG scintigraphy and/or persistence of tumour on bone marrow aspirate and trephine morphological examination), mixed response (MR), stable disease (SD), or progressive disease (PD) according to the International Neuroblastoma Response Criteria (INRC) [9].

Other eligibility criteria included a absolute neutrophil count $($ ANC) $>1,000 / \mu \mathrm{L}$, platelet count $>100,000 / \mu \mathrm{L}$, creatinine level $<1.5 \mathrm{mg} / \mathrm{dL}$, total bilirubin $<1.2 \mathrm{mg} / \mathrm{dL}$, aspartate aminotransferase, and alanine aminotransferase $<2.5 \mathrm{SD}$ of the reference laboratory, and normal cardiac function on echocardiography. Exclusion criteria included any severe organ dysfunction, known human immunodeficiency virus infection, hepatitis or hepatitis $C$ virus infection and previous treatment with doxorubicin or other treatment besides the COJEC regimen.

According to the TVD regimen, topotecan was administered intravenously as a 30 -minute infusion in $0.9 \%$ saline in $100 \mathrm{~mL} / \mathrm{m}^{2}$ and a dose of $1.5 \mathrm{mg} / \mathrm{m}^{2} /$ day for 5 consecutive days. Vincristine was administered in a 48-hour continuous intravenous infusion at a dose of $1 \mathrm{mg} / \mathrm{m}^{2} /$ day in $125 \mathrm{~mL} /$ $\mathrm{m}^{2} /$ day $0.9 \%$ saline (maximum dose, $1 \mathrm{mg} /$ day), starting 1 hour after the final topotecan infusion. Doxorubicin was administered intravenously simultaneously with vincristine at a dose of $22.5 \mathrm{mg} / \mathrm{m}^{2} /$ day in $125 \mathrm{~mL} / \mathrm{m}^{2} /$ day of $0.9 \%$ saline solution. Granulocyte-colony stimulating factor (filgrastim) was administered at a dose of $5 \mu \mathrm{g} / \mathrm{kg} /$ day subcutaneously starting 48 hours after the end of the TVD course and continuing until neutrophil recovery (ANC $>2,500 / \mu \mathrm{L}$ ). Anti-emetic therapy was administered according to institutional policies.

The second course of TVD was scheduled 21-28 days after completion of the first course, following hematologic recovery $(\mathrm{ANC}>1,000 / \mu \mathrm{L}$, platelet count $>100,000 / \mu \mathrm{L})$, in the absence of clinical evidence of PD and nonhematological toxicity greater than grade 1 , according to the Common Terminology Criteria for Adverse Events (http:// ctep.cancer. gov). Febrile episodes were classified according to standard international criteria [10].

After completion of the second TVD course, the overall and metastatic tumour response were evaluated by means of a computed tomography or magnetic resonance imaging scan, mIBG scintigraphy, morphological examination of bone marrow aspirates and trephines from at least two sites according to the INRC criteria. However, according to the SIOPEN HR-NBL-1 protocol, only patients achieving metastatic CR or GPR were eligible for HDT. All other patients 


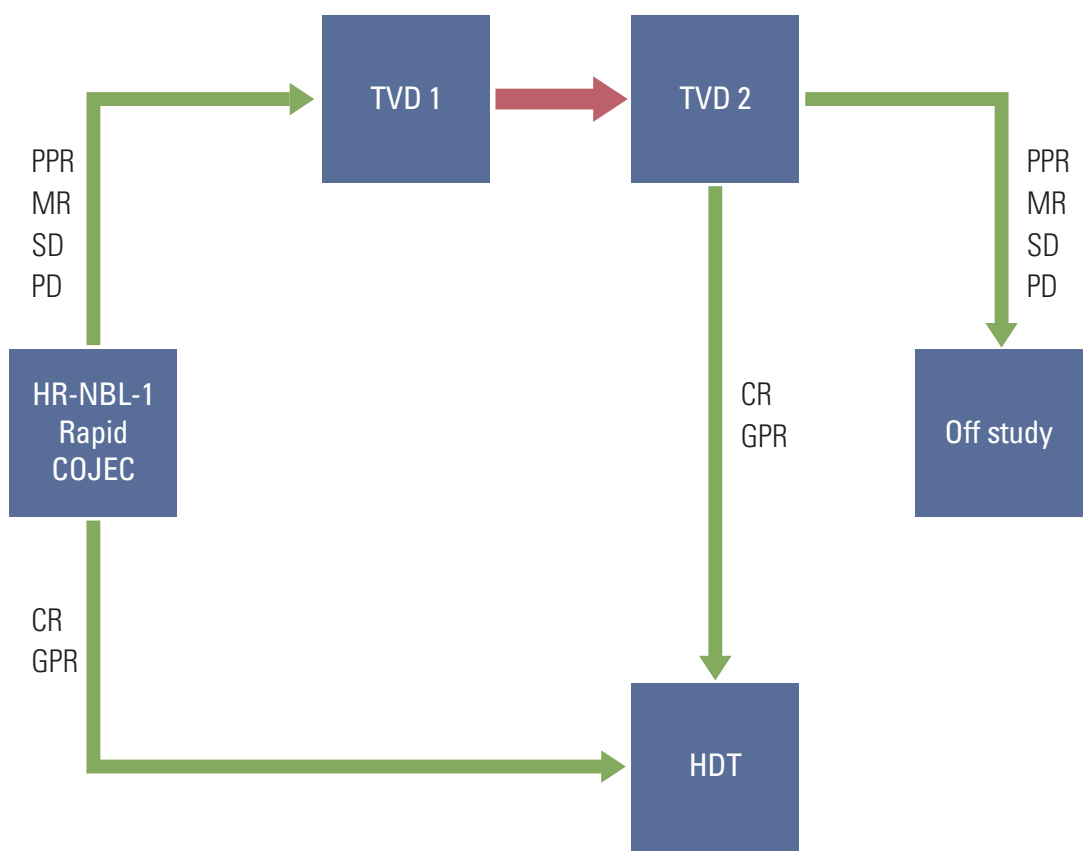

Fig. 1. The topotecan-vincristine-doxorubicin (TVD) salvage therapy for children included in the HR-NBL-1 protocol. Patients with persistent metastatic disease after the COJEC induction therapy of the International Society of Paediatric Oncology Europe Neuroblastoma (SIOPEN) HR-NBL-1 protocol were eligible for TVD therapy with the aim of eradicating the metastatic disease. Children achieving complete remission (CR) or good partial response (GPR) become eligible for consolidation with high dose therapy (HDT) as planned by the original HR-NBL-1 protocol, all the other were considered failures. PPR, poor partial response; $\mathrm{MR}$, mixed response; $\mathrm{SD}$, stable disease; $\mathrm{PD}$, progressive disease.

became eligible for any other salvage treatment (Fig. 1).

Surgical resection of the primary tumour was recommended before HDT, if possible.

The study was approved by national or institutional ethics committees and legal guardians and/or participants were required to provide informed consent prior participation.

\section{Statistical methods}

For this phase II study, the primary endpoint was the RR after two courses of TVD. Achievement of either a CR, or any PR according to the INRC [9] after two courses of TVD was considered a success, while PD, SD, and MR were considered failures.

The study had a two-stage design according to Simon [11], with an accepted $\alpha$ error of 0.05 and a power of $90 \%$. In this model, a RR $<30 \%$ was not considered as interesting (P0), while a $R R \geq 50 \%$ was considered sufficient to accept the treatment for further study. According to this design, it was necessary to recruit 24 patients in the first stage, and if at least eight responses (CR or PR) were observed, the study could continue to a total of 63 enrolled patients.
The percentage of patients who achieved the SIOPEN criteria to proceed to HDT and the response of metastases according to metastatic status at study entry were also calculated.

Binomial exact confidence intervals $(95 \% \mathrm{CI})$ were calculated and reported for all response and toxicity rates. The chi squared test or Fisher exact test, when applicable, were used to compare categorical variables.

\section{Results}

\section{Baseline characteristics}

From April 2007 to October 2009, a total of 65 patients from six European countries (30 centers) were enrolled into the study; two of whom were subsequently excluded (one because of stage 3 disease at diagnosis, one for insufficient data). The characteristics of the remaining 63 (40 males, 23 females) eligible and evaluable patients are summarised in Table 1 . The 
Table 1. Characteristics at diagnosis and at TVD therapy of 63 patients with refractory metastatic neuroblastoma after COJEC induction

\begin{tabular}{|c|c|}
\hline Characteristic & No. $(\%)$ \\
\hline \multicolumn{2}{|l|}{ At diagnosis } \\
\hline Age, median (IQR, mo) & $46(28-69)$ \\
\hline \multicolumn{2}{|l|}{ Primary tumour site } \\
\hline Abdomen & $55(87.3)$ \\
\hline Chest & $6(9.5)$ \\
\hline Other & $2(3.2)$ \\
\hline Stage 4 & $63(100)$ \\
\hline MYCN, amplified ${ }^{\text {a) }}$ & $13(23.6)$ \\
\hline \multicolumn{2}{|l|}{ At study entry } \\
\hline \multicolumn{2}{|l|}{ Overall INRC response post-COJEC } \\
\hline PR & $40(63.52)$ \\
\hline MR & $7(11.1)$ \\
\hline SD & $15(23.8)$ \\
\hline PD & $1(1.6)$ \\
\hline \multicolumn{2}{|l|}{ Metastatic response post-COJEC } \\
\hline PR & $46(73.0)$ \\
\hline SD & $16(25.4)$ \\
\hline PD & $1(1.6)$ \\
\hline \multicolumn{2}{|l|}{$\begin{array}{l}\text { Localization of metastatic disease } \\
\text { at TVD entry }\end{array}$} \\
\hline Bone marrow only & $10(15.9)^{b)}$ \\
\hline Skeleton only & $\left.25(39.7)^{c}\right)$ \\
\hline Combined bone marrow and skeleton & $27(42.9)^{b), c)}$ \\
\hline Liver & $1(1.6)$ \\
\hline
\end{tabular}

TVD, topotecan-vincristine-doxorubicin; IQR, interquartile range; INRC, International Neuroblastoma Response Criteria; $\mathrm{PR}$, partial response; $\mathrm{MR}$, mixed response; $\mathrm{SD}$, stable disease; $\mathrm{PD}$, progressive disease; $\mathrm{mIBG}$, metaiodobenzylguanidine. ${ }^{\mathrm{a}}$ Based on 55 evaluable cases, ${ }^{\mathrm{b}}$ Total bone marrow positive $(\mathrm{n}=37)$, ${ }^{\mathrm{c}}$ Total $\mathrm{mIBG}$ skeleton positive $(\mathrm{n}=52)$.

overall response according to INRC at study entry (i.e., after rapid COJEC) was PPR in 40 patients (63.5\%), MR in seven (11.1\%) patients, SD in $15(23.8 \%)$ patients, and PD in one patient $(1.6 \%)$. Of the seven patients with an overall MR according to INRC, six had a metastatic PPR and one had metastatic SD. Thus, if only the metastatic response was considered, 46 children (73\%) were in PPR, 16 (25.4\%) had SD and one $(1.6 \%)$ had PD. With regard to the metastatic sites, 37 patients $(58.7 \%)$ had bone marrow involvement, 52 (82.5\%) had skeletal mIBG-positive metastatic disease and only one $(1.6 \%)$ had liver metastases (Table 2).

\section{Antitumor activity}

According to the statistical design, after enrollment of the first 24 evaluable patients, 10 positive responses (1 CR, 9 PR) were documented; thus, 39 additional patients were recruited to complete the study.

\section{1) Overall response (including primary tumour) according to INRC}

Following two courses of TVD, four patients (6.4\%) achieved a CR and 28 any PR (44.4\%), for an overall RR of $50.8 \%$ (95\% CI, 37.9 to 63.6). Of the remaining patients, 16 had a MR (25.4\%); 14, a SD (22.2\%); and 1, a PD (1.6\%).

\section{2) Achievement of SIOPEN HR-NBL criteria to proceed to HDT}

In addition to the four patients who achieved an overall CR, 14 of the 28 children achieved an overall PR, 10 achieved a metastatic CR and four GPR. Moreover, of the 16 children with an overall MR, three had a metastatic CR and two a GPR. Therefore, 23 patients (36.5\%; 95\% CI, 24.7 to 49.6 ; 17 CR and 6 GPR with $\leq 3$ mIBG skeletal areas and no bone marrow disease) were eligible to proceed to HDT according to the SIOPEN criteria.

Table 3 reports on the metastatic response after TVD stratified by metastatic response after rapid COJEC. Of the 46 children who entered the study with a PR after rapid COJEC, 20 (43.4\%; 14 in CR and six in PR with $\leq 3$ mIBG spots) became eligible for HDT, as compared to only three of the 17 who entered the study (18.8\%) with either SD or PD, $\mathrm{p}=0.059$.

The response after TVD is reported in Table 2 after stratification by site of metastatic disease. Overall, seven CR (70\%) were documented in the 10 patients with only bone marrow disease, five CR and six PR with $\leq 3 \mathrm{mIBG}$ skeletal spots (44\%) were documented in the 25 children with only mIBG-positive scan, five CR (18.5\%) were documented among the 27 patients with combined positive metastatic disease, and one SD was documented in the only child with hepatic disease. The difference among groups was statistically significant $(\mathrm{p}=0.012$, Fisher exact test). If the metastatic sites were analyzed separately, 17 of the 37 positive bone marrows (45.9\%) cleared after TVD, while only 10 of the 52 skeletal metastases (19.2\%) achieved CR ( $\mathrm{p}=0.007)$.

MYCN status did not affect the probability of positive response to TVD. In fact, among the 55 subjects assessed for MYCN, the 13 MYCN amplified patients had a RR (CR or any PR) similar to that of the 42 not amplified (8 [61.5\%] vs. 20 [47.6\%]; $\mathrm{p}=0.38$, chi-square test). Similarly, six MYCN amplified $(46.1 \%)$ became eligible for HDT, while versus 15 of the not amplified patients $(35.7 \%)(\mathrm{p}=0.53$, Fisher exact test). 
Table 2. Metastatic response after two courses of TVD by site of metastatic disease at study entry

\begin{tabular}{|c|c|c|c|c|c|c|c|}
\hline \multirow[b]{3}{*}{ Site of metastatic disease } & & \multicolumn{6}{|c|}{ Metastatic response after TVD } \\
\hline & & \multicolumn{2}{|c|}{ Eligible to HDT } & \multicolumn{4}{|c|}{ Not eligible to HDT } \\
\hline & & sp & $\begin{array}{c}\mathrm{PR} \leq 3 \mathrm{mIBG} \\
\text { spots and negative } \\
\text { bone marrow }\end{array}$ & $\begin{array}{c}\text { PR }>3 \text { mIBG } \\
\text { spots and negative } \\
\text { bone marrow }\end{array}$ & MR & SD & PD \\
\hline Bone marrow only & 10 & $7(70.0)^{\mathrm{a})}$ & 0 & 0 & 0 & $3(30.0)$ & 0 \\
\hline Skeleton only & 25 & $5(20.0)^{\mathrm{b})}$ & $6(24.0)$ & $4(16.0)$ & 0 & $10(40.0)$ & 0 \\
\hline $\begin{array}{l}\text { Combined bone marrow } \\
\text { and skeleton }\end{array}$ & 27 & $5(18.5)^{\mathrm{a}), \mathrm{b}}$ & 0 & $\left.5(18.5)^{a}\right)$ & $16(59.3)$ & 0 & $1(3.7)$ \\
\hline Liver & 1 & 0 & 0 & 0 & 0 & $1(100)$ & 0 \\
\hline Total & 63 & $17(27.0)$ & $6(9.5)$ & $9(14.3)$ & $16(25.4)$ & $14(22.2)$ & $1(1.6)$ \\
\hline
\end{tabular}

Values are presented as number (\%). Eligibility to HDT: $\mathrm{p}=0.012$ (Fisher exact test). TVD, topotecan-vincristine-doxorubicin; $\mathrm{HDT}$, high dose therapy; $\mathrm{CR}$, complete response; $\mathrm{mIBG}$, metaiodobenzylguanidine; $\mathrm{PR}$, partial response; $\mathrm{MR}$, mixed response; $\mathrm{SD}$, stable disease; $\mathrm{PD}$, progressive disease. ${ }^{\mathrm{a}} \mathrm{BM}$ cleared, ${ }^{\mathrm{b}}$ Skeleton cleared.

Table 3. Metastatic response after TVD stratified by metastatic response after COJEC

\begin{tabular}{|c|c|c|c|c|c|c|c|}
\hline \multirow[b]{2}{*}{$\begin{array}{l}\text { Metastatic response } \\
\text { after COJEC }\end{array}$} & & \multicolumn{6}{|c|}{ Metastatic response after TVD } \\
\hline & & CR & $\begin{array}{c}\mathrm{PR} \leq 3 \mathrm{mIBG} \\
\text { spots and negative } \\
\text { bone marrow }\end{array}$ & $\begin{array}{c}\text { PR }>3 \text { mIBG } \\
\text { spots and negative } \\
\text { bone marrow }\end{array}$ & MR & SD & PD \\
\hline PR & $46(73.0)$ & $14(30.4)$ & $6(13.0)$ & $9(21.7)$ & $10(21.7)$ & 7 (15.2) & 0 \\
\hline SD & $16(25.4)$ & $3(18.8)$ & 0 & 0 & $6(37.5)$ & $7(43.7)$ & 0 \\
\hline PD & $1(1.6)$ & 0 & 0 & 0 & 0 & 0 & $1(100)$ \\
\hline Total & 63 & $17(27.0)$ & $6(9.5)$ & 9 (14.3) & $16(25.4)$ & $14(22.2)$ & $1(1.6)$ \\
\hline
\end{tabular}

Values are presented as number (\%). Eligibility to HDT: $\mathrm{p}=0.059$ (chi-square test) comparing patients with PR after COJEC vs. those with $<\mathrm{PR}$. TVD, topotecan-vincristine-doxorubicin; $\mathrm{CR}$, complete response; PR, partial response; $\mathrm{mIBG}$, metaiodobenzylguanidine; $\mathrm{MR}$, mixed response; $\mathrm{SD}$, stable disease; $\mathrm{PD}$, progressive disease.

Table 4. Grade 3-4 toxicity after 126 TVD courses in 63 patients

\begin{tabular}{|c|c|c|c|c|}
\hline Toxicity & 1st course & 2nd course & Overall & Patients \\
\hline \multicolumn{5}{|l|}{ Hematologic } \\
\hline Neutropenia & $55(87.3)$ & $51(80.9)$ & $106(84.1)$ & $57(90.5)$ \\
\hline Thrombocytopenia & $54(85.7)$ & $50(79.4)$ & $104(82.5)$ & $58(92.1)$ \\
\hline Anaemia & $40(63.5)$ & $37(58.7)$ & $77(61.1)$ & $48(76.2)$ \\
\hline \multicolumn{5}{|l|}{ Nonhematologic } \\
\hline Fever $>38^{\circ} \mathrm{C}$ & $29(46.0)$ & $24(38.1)$ & $53(42.1)$ & $38(60.3)$ \\
\hline Mucositis & $17(27.0)$ & $8(12.7)$ & $25(19.8)$ & $20(31.7)$ \\
\hline Vomiting & $2(3.2)$ & $1(1.3)$ & $3(2.4)$ & $3(4.8)$ \\
\hline Constipation & $1(1.6)$ & $0(0.0)$ & $1(0.8)$ & $1(1.6)$ \\
\hline Sensory neuropathy & $2(3.2)$ & $1(1.6)$ & $3(2.4)$ & $2(3.2)$ \\
\hline
\end{tabular}

Values are presented as number (\%). TVD, topotecan-vincristine-doxorubicin. 
No difference in achieving the eligibility to HDT was observed after stratification of patients according to MYCN status of their primary tumour.

\section{Toxicity}

All patients received the two planned TVD courses for a total of 126 evaluable courses. No chemotherapy-related toxic deaths were reported, and the observed toxicity was mostly haematological (Table 4). Overall, 106 courses $(84.1 \%$; $95 \%$ CI, 76.5 to 90.0$)$ were complicated by grade 3 or 4 neutropenia; $104(82.5 \%$; $95 \%$ CI, 74.8 to 88.7$)$ by grade 3 or 4 thrombocytopenia and $77(61.1 \%$; 95\% CI, 52.0 to 69.7$)$ by grade 3 or 4 anaemia. Slightly more toxicities were observed after the first course (not significant). Only six (9.5\%), five (7.9\%), $15(23.8 \%)$, and four $(6.4 \%)$ patients showed no evidence of neutropenia, thrombocytopenia, anaemia or any haematological toxicity, respectively.

Fever was the most frequently reported non-haematological complication documented after 53 courses $(42.1 \%$; 95\% CI, 33.3 to 51.2). Hospitalization for systemic antibiotic therapy was required following 43 courses $(34.1 \%$; $95 \%$ CI, 25.9 to 43.1$) ; 25$ $(39.7 \%$; $95 \%$ CI, 27.6 to 52.8$)$ after the first and 18 (28.6\%; $95 \%$ CI, 17.9 to 41.3$)$ after the second course. Most episodes of fever (48 [90.6\%; 95\% CI, 79.3 to 97.9]) were classified as fever of unknown origin, and only five $(9.4 \%$; 95\% CI, 3.1 to 20.7$)$ as documented infection (with one case of bacteraemia that was central venous catheter-related). Stomatitis was the second most common nonhaematological toxicity occurring after 25 courses $(19.8 \%$; 95\% CI, 13.3 to 27.9$)$ in 20 patients $(31.7 \%)$. Severe vomiting (grade 3 or 4 ) was reported after three courses $(2.4 \%$; $95 \%$ CI, 0.5 to 6.8$)$ in three patients, and severe sensory neuropathy occurred after three courses $(3.2 \%$; 95\% CI, 0.5 to $6.8)$ in two patients. Only one case $(0.8 \%$; $95 \%$ CI, 0.02 to 4.3$)$ of grade 4 constipation was documented.

A 30\% dose reduction of the second TVD course was scheduled for the patient with grade 4 constipation. There was a $25 \%$ dose reduction in three of the 25 patients with severe stomatitis, and vincristine was only reduced by $50 \%$ in two children with severe neuropathy after the first course of TVD.

\section{Discussion}

This study provides further evidence of the efficacy of TVD in metastatic neuroblastoma failing to achieve a metastatic CR after rapid COJEC (which does not include topotecan or doxorubicin) according to the SIOPEN HR-NBL-1 protocol. A previous Italian phase II study demonstrated that this combination is effective for treatment of refractory and relapsed (stage 3 and 4) disease, leading to a combined $64 \%$ CR and PR RR [8]. The TVD combination was designed based on pre-clinical studies demonstrating that topoisomerase I (campothecins) and II (doxorubicin) inhibitors, if administered in this sequence, have synergistic effects without increased toxicity. In addition, the therapeutic effects of combining topotecan with vincristine were greater than the additive effect of the agents alone with moderate toxicity [12-14]. In the current study, we showed that two courses of TVD improved the response of $36.5 \%$ of patients with highrisk neuroblastoma who failed to achieve the SIOPEN HRNBL criteria after first-line therapy with rapid COJEC, and that these patients are then eligible to proceed to HDT.

In our series, the bone marrow was more likely to be cleared of tumour burden than the skeletal disease. Similarly, patients who entered TVD after having already demonstrated some response to COJEC tended to have a better RR than those who entered TVD after having experienced only SD or PD.

The results reported herein are not fully comparable with those from previous studies that also used topotecan in highrisk neuroblastoma, mainly because of differences in patient selection and schedule of drug administration. In particular, this study only comprised patients with PR, MR, SD, or PD after first-line therapy, while most other studies included patients with both relapsed and refractory disease [15-23]. These previous studies generally reported small and heterogeneous series that received topotecan either alone [15-19] with response rates ranging from $<20 \%$ to $60 \%$, or in combination with other drugs including cyclophosphamide $[19,20]$, temozolomide [21,22], or cyclophosphamide-etoposide [23] with response rates ranging from $32 \%$ to $64 \%$.

To the best of our knowledge, only two other studies have focused on previously untreated neuroblastoma patients in which topotecan was administered in combination with cyclophosphamide at two different dosages, and the reported response rates were $76 \%$ [24] and 84\% [25], respectively.

TVD is a manageable therapeutic regimen. In our study, no toxic deaths were observed and schedule reduction was only necessary in six subjects. Myelosuppression was the main treatment-related toxicity. Although hospitalisation for systemic antibiotic therapy was required after $34 \%$ of the courses, documented infection was only reported in five patients.

In conclusion, this multi-centre European phase II study demonstrated that the combination of topotecan, vincristine and doxorubicin increased the RR in $36.5 \%$ of patients with persistent or refractory high-risk neuroblastoma disease when following an intensive multi-agent induction chemotherapy that does not include topotecan or doxorubicin. Children with only bone marrow disease and those who had already shown some chemo-sensitivity to induction therapy 
are more likely to benefit from TVD.

Based on the results of this study and because doxorubicin and topotecan are not included in the COJEC regimen, we conclude that the TVD regimen has a role in improving the RR to COJEC induction therapy and thereby in increasing the number of patients eligible for HDT according to the SIOPEN HR-NBL-1 protocol criteria. The long term benefits of TVD compared to other first line chemotherapy combinations need to be determined in randomized clinical trials.

\section{Conflicts of Interest}

Conflict of interest relevant to this article was not reported.

\section{Acknowledgments}

L. Amoroso and G. Erminio are funded by the Italian Neuroblastoma Foundation. A. D. J. Pearson is funded through a Cancer Research UK Life Chair and Programme Grant included within a Cancer Research UK ICR Core Award (C347/A15403) and is supported by the NIHRRM/ICR Biomedical Research Centre.

\section{Author Details}

${ }^{1}$ Paediatric Oncology, Istituto Giannina Gaslini, Genova, ${ }^{2}$ Epidemiology and Biostatistics Unit, Istituto Giannina Gaslini, Genova, Italy, ${ }^{3}$ Institute of Cancer Sciences, Manchester Cancer Research Centre, Manchester Academic Health Sciences Centre, University of Manchester, Manchester, ${ }^{4}$ Divisions of Cancer Therapeutics and Clinical Studies, Institute of Cancer Research and Children and Young People's Unit, The Royal Marsden NHS Foundation Trust, London, ${ }^{5}$ Paediatric Oncology, Great Ormond Street Hospital for Children NHS Foundation Trust, London, UK, ${ }^{\circ}$ Paediatric Oncology, Institute Gustave Roussy, Villejuif, France, ${ }^{7}$ Paediatric Oncology, Hospital Universitario La Fe, Valencia, Spain, ${ }^{8}$ Department of HematologyOncology Hopital des Enfants, Toulouse, France, ${ }^{9}$ Department of Paediatric Hematology, Oncology and Stem Cell Transplantation, Ghent University Hospital, Ghent, Belgium, ${ }^{10}$ Pediatric Intensive Care and Onco-Hematology Units, Nantes Hospital, Nantes, France, ${ }^{11}$ Department of Paediatric Oncology, Istituto Nazionale Tumori, Milan, Italy, ${ }^{12}$ Children's Cancer Research Institute, St. Anna Children's Hospital, Vienna, Austria

\section{References}

1. Haupt R, Garaventa A, Gambini C, Parodi S, Cangemi G, Casale $\mathrm{F}$, et al. Improved survival of children with neuroblastoma between 1979 and 2005: a report of the Italian Neuroblastoma Registry. J Clin Oncol. 2010;28:2331-8.

2. Kreissman SG, Seeger RC, Matthay KK, London WB, Sposto R, Grupp SA, et al. Purged versus non-purged peripheral blood stem-cell transplantation for high-risk neuroblastoma (COG A3973): a randomised phase 3 trial. Lancet Oncol. 2013;14:999-1008.

3. Pearson AD, Pinkerton CR, Lewis IJ, Imeson J, Ellershaw C, Machin D, et al. High-dose rapid and standard induction chemo-therapy for patients aged over 1 year with stage 4 neuroblastoma: a randomised trial. Lancet Oncol. 2008;9:247-56.

4. Ladenstein R, Valteau-Couanet D, Brock P, Yaniv I, Castel V, Laureys $\mathrm{G}$, et al. Randomized Trial of prophylactic granulocyte colony-stimulating factor during rapid COJEC induction in pediatric patients with high-risk neuroblastoma: the European HR-NBL1/SIOPEN study. J Clin Oncol. 2010;28:3516-24.

5. Ladenstein R, Potschger U, Hartman O, Pearson AD, Klingebiel T, Castel V, et al. 28 years of high-dose therapy and SCT for neuroblastoma in Europe: lessons from more than 4000 procedures. Bone Marrow Transplant. 2008;41 Suppl 2: S118-27.

6. Decarolis B, Schneider C, Hero B, Simon T, Volland R, Roels $\mathrm{F}$, et al. Iodine-123 metaiodobenzylguanidine scintigraphy scoring allows prediction of outcome in patients with stage 4 neuroblastoma: results of the Cologne interscore comparison study. J Clin Oncol. 2013;31:944-51.

7. Yanik GA, Parisi MT, Shulkin BL, Naranjo A, Kreissman SG, London WB, et al. Semiquantitative $\mathrm{mIBG}$ scoring as a prognostic indicator in patients with stage 4 neuroblastoma: a report from the Children's oncology group. J Nucl Med. 2013;54:541-8.

8. Garaventa A, Luksch R, Biasotti S, Severi G, Pizzitola MR, Viscardi $\mathrm{E}$, et al. A phase II study of topotecan with vincristine and doxorubicin in children with recurrent/ refractory neuroblastoma. Cancer. 2003;98:2488-94.

9. Brodeur GM, Pritchard J, Berthold F, Carlsen NL, Castel V, Castelberry RP, et al. Revisions of the international criteria for neuroblastoma diagnosis, staging, and response to treatment. J Clin Oncol. 1993;11:1466-77.

10. Castagnola E, Mikulska M, Viscoli C. Prophylaxis and empirical therapy of infection in cancer patients. In: Bennett JE, Dolin R, Blaser MJ, editors. Mandell, Douglas, and Bennett's principles and practice of infectious diseases. New York: Elsevier; 2015. p. 3395-413.

11. Simon R. Optimal two-stage designs for phase II clinical trials. Control Clin Trials. 1989;10:1-10.

12. Kim JH, Kim SH, Kolozsvary A, Khil MS. Potentiation of radiation response in human carcinoma cells in vitro and murine fibrosarcoma in vivo by topotecan, an inhibitor of DNA topoisomerase I. Int J Radiat Oncol Biol Phys. 1992;22: 
515-8.

13. Rowinsky EK, Verweij J. Review of phase I clinical studies with topotecan. Semin Oncol. 1997;24(6 Suppl 20):S20-3S20-10.

14. Thompson J, George EO, Poquette CA, Cheshire PJ, Richmond LB, de Graaf SS, et al. Synergy of topotecan in combination with vincristine for treatment of pediatric solid tumor xenografts. Clin Cancer Res. 1999;5:3617-31.

15. Vassal G, Pondarre C, Cappelli C, Terrier-Lacombe MJ, Boland I, Morizet J, et al. DNA-topoisomerase I, a new target for the treatment of neuroblastoma. Eur J Cancer. 1997;33:2011-5.

16. Pratt CB, Stewart C, Santana VM, Bowman L, Furman W, Ochs J, et al. Phase I study of topotecan for pediatric patients with malignant solid tumors. J Clin Oncol. 1994;12:539-43.

17. Blaney SM, Needle MN, Gillespie A, Sato JK, Reaman GH, Berg SL, et al. Phase II trial of topotecan administered as 72hour continuous infusion in children with refractory solid tumors: a collaborative Pediatric Branch, National Cancer Institute, and Children's Cancer Group Study. Clin Cancer Res. 1998;4:357-60.

18. Santana VM, Furman WL, Billups CA, Hoffer F, Davidoff AM, Houghton PJ, et al. Improved response in high-risk neuroblastoma with protracted topotecan administration using a pharmacokinetically guided dosing approach. J Clin Oncol. 2005;23:4039-47.

19. London WB, Frantz CN, Campbell LA, Seeger RC, Brumback BA, Cohn SL, et al. Phase II randomized comparison of topotecan plus cyclophosphamide versus topotecan alone in children with recurrent or refractory neuroblastoma: a Children's Oncology Group study. J Clin Oncol. 2010;28:3808-15.
20. Saylors RL 3rd, Stine KC, Sullivan J, Kepner JL, Wall DA, Bernstein ML, et al. Cyclophosphamide plus topotecan in children with recurrent or refractory solid tumors: a Pediatric Oncology Group phase II study. J Clin Oncol. 2001;19:3463-9.

21. Rubie H, Geoerger B, Frappaz D, Schmitt A, Leblond P, Ndiaye $\mathrm{A}$, et al. Phase I study of topotecan in combination with temozolomide (TOTEM) in relapsed or refractory paediatric solid tumours. Eur J Cancer. 2010;46:2763-70.

22. Di Giannatale A, Dias-Gastellier N, Devos A, Mc Hugh K, Boubaker A, Courbon F, et al. Phase II study of temozolomide in combination with topotecan (TOTEM) in relapsed or refractory neuroblastoma: a European Innovative Therapies for Children with Cancer-SIOP-European Neuroblastoma study. Eur J Cancer. 2014;50:170-7.

23. Simon T, Langler A, Harnischmacher U, Fruhwald MC, Jorch N, Claviez A, et al. Topotecan, cyclophosphamide, and etoposide (TCE) in the treatment of high-risk neuroblastoma. Results of a phase-II trial. J Cancer Res Clin Oncol. 2007;133: 653-61.

24. Kretschmar CS, Kletzel M, Murray K, Thorner P, Joshi V, Marcus $\mathrm{R}$, et al. Response to paclitaxel, topotecan, and topotecancyclophosphamide in children with untreated disseminated neuroblastoma treated in an upfront phase II investigational window: a pediatric oncology group study. J Clin Oncol. 2004;22:4119-26.

25. Park JR, Scott JR, Stewart CF, London WB, Naranjo A, Santana $\mathrm{VM}$, et al. Pilot induction regimen incorporating pharmacokinetically guided topotecan for treatment of newly diagnosed high-risk neuroblastoma: a Children's Oncology Group study. J Clin Oncol. 2011;29:4351-7. 